\title{
Polymer Optical Fibre Sensors for Endoscopic Opto-Acoustic Imaging
}

Christian Broadway ${ }^{1}$, Daniel Gallego ${ }^{1}$, Getinet Woyessa ${ }^{3}$, Andreas Pospori²,Ole Bang ${ }^{3}$, David J

$\mathrm{Webb}^{2}$, Guillermo Carpintero ${ }^{1}$, Horacio Lamela ${ }^{1}$

${ }^{1}$ Opto-Electronics and Laser Technology Group (GOTL), Universidad Carlos III de Madrid, ${ }^{2}$ Aston

Institute of Photonic Technologies, Aston University, ${ }^{3}$ DTU Fotonik, Denmark Technical University

\begin{abstract}
Opto-acoustic imaging (OAI) shows particular promise for in-vivo biomedical diagnostics. Its applications include cardiovascular, gastrointestinal and urogenital systems imaging. Opto-acoustic endoscopy (OAE) allows the imaging of body parts through cavities permitting entry. The critical parameter is the physical size of the device, allowing compatibility with current technology, while governing flexibility of the distal end of the endoscope based on the needs of the sensor. Polymer optical fibre (POF) presents a novel approach for endoscopic applications and has been positively discussed and compared in existing publications. A great advantage can be obtained for endoscopy due to a small size and array potential to provide discrete imaging speed improvements. Optical fibre exhibits numerous advantages over conventional piezo-electric transducers, such as immunity from electromagnetic interference and a higher resolution at small sizes. Furthermore, micro structured polymer optical fibres offer over 12 times the sensitivity of silica fibre. We present a polymer fibre Bragg grating ultrasound detector with a core diameter of 125 microns. We discuss the ultrasonic signals received and draw conclusions on the opportunities and challenges of applying this technology in biomedical applications.
\end{abstract}

Keywords: Polymer Optical Fibre Sensors, Fibre Sensors, Polymer Optical Fibre, Endoscopy, Biomedical Applications , Opto-Acoustic Imaging

\section{INTRODUCTION}

Opto-acoustic imaging is a growing field of research in recent years, providing functional imaging of physiological parameters such as haemoglobin concentration. Opto-acoustic imaging provides the benefit relatively speckle free imaging with a high contrast at high ultrasonic resolution [1]. Opto-acoustic detection re quires ultra wideband ultrasonic sensors, as a typical opto-acoustic frequency spectrum ranges from $\sim 100 \mathrm{kHz}$ to $\sim 50 \mathrm{MHz}$.

Endoscopy provides a means by which opto-acoustic imaging can be profitably used. Jenson et al established the need for a target endoscopic probe diameter of less than $1 \mathrm{~mm}$ for clinical imaging, which has not yet been achieved to the best of our knowledge [2]. Recent advances include a $2.5 \mathrm{~mm}$ diameter dual-mode endoscope by Yang et al in 2012 [3], followed by a $1.1 \mathrm{~mm}$ probe by Bai et al in 2014 [4]. The latter submission discusses the relationship between size and sensitivity of piezo-electric transducers, an important issue given the target diameter and the widespread promulgation of piezo-electric transducers as detectors.

Piezo-electric transducers are highly sensitive, but have several failings. Key points for consideration are; their sensitivity being proportional to size, limited bandwidth and a susceptibility to electromagnetic interference [5]. These issues are inherently tied to the nature of piezo electric transducers. Opto-Acoustic endoscopy requires a wide bandwidth and small detector size within a small and confined environment, where a large proportion of the probe may be taken up by scanning mechanisms (such as an acoustic mirror and micro-motor).

Our group is developing polymer optical fibre technology for opto-acoustic endoscopy as a way to better fulfil the requirements previously stated and to get closer to the $1 \mathrm{~mm}$ target diameter. Polymer optical fibre (POF) delivers improved mechanical properties and a significant potential that can theoretically meet the requirements for compactness, sensitivity and bandwidth. However, POF has a small number of disadvantages, most of which are related to the newness of the technology and limited commercialisation.

Silica fibre and POF are electromagnetically insensitive and can provide a wide and customizable bandwidth for sensing, especially when using intrinsic fibre structures. It is important to note, however, the important advantages that POF has over silica. While silica is commercially available and indeed mature, it is nonetheless a brittle material that would be

*cbroadwa@ing.uc3m.es; phone +34 624 8758; portal.uc3m.es/portal/page/portal/grupos_investigacion/optoelectronics

Opto-Acoustic Methods and Applications in Biophotonics II, edited by

Vasilis Ntziachristos, Roger Zemp, Proc. of SPIE-OSA Vol. 9539, 953907

(C) 2015 SPIE-OSA · CCC code: $1605-7422 / 15 / \$ 18 \cdot$ doi: $10.1117 / 12.2183452$

Proc. of SPIE Vol. $9539953907-1$ 
placed inside the body, albeit with protection. The mechanical properties of POF not only reduce safety concerns but also present the potential for greater sensitivity. It is important to note that several polymers exist under the banner of polymer optical fibre, the most common of which being Poly-Methyl-Methacrylate (PMMA). Bilro et al give the Young's Modulus of PMMA as 3.2GPa, compared to 72GPa for Silica [6]. Furthermore, Peters lists the elastic limit of PMMA as $10 \%$ compared to $3 \%$ for silica [7]. These qualities not only show higher potential for acoustic sensitivity but also highlight the reduced risk of fracture and bending for POF compared to silica. While these figures may vary for different variations of the polymer PMMA and indeed for other polymers, it shows the scope and potential that POF offers for endoscopic implementation.

We have previously demonstrated that the acoustic sensitivity of an interferometric single mode polymer optical fibre sensor (SMPOF) is one order of magnitude higher than a silica counterpart [8]. However, good quality step index SMPOF is not easily available at the present time. Micro-structured polymer optical fibre (mPOF) exhibits the same acoustic sensitivity at $1 \mathrm{MHz}$, can be made endlessly single-mode and is 3 times more sensitive at $10 \mathrm{MHz}$ [9].

Intrinsic fibre structures, such as fibre Bragg gratings (FBG) and Fabry-Perot cavities, are inscribed into optical fibre and behave according to the forces that act on it, such as strain and temperature. Intrinsic structures have a customisable optical spectral response and can be highly reflective. Factors such as birefringence and fibre geometry affect the normally homogeneous intrinsic structures that can be observed in silica fibre, rendering their implementation more difficult.

In this contribution, we present an mPOF PMMA Bragg grating detector with a $125 \mu \mathrm{m}$ diameter, making it suitable for endoscopic implementation. We demonstrate ultrasonic detection at $1 \mathrm{MHz}$ and $10 \mathrm{MHz}$. We analyse the different signals received and identify the causes, presenting plans for the removal of unwanted components in order to improve the detector. We relate the various components to endoscopic sensor configuration and topologies. Finally, we discuss the potential for opto-acoustic imaging and barriers that need to be overcome.

\section{METHODOLOGY}
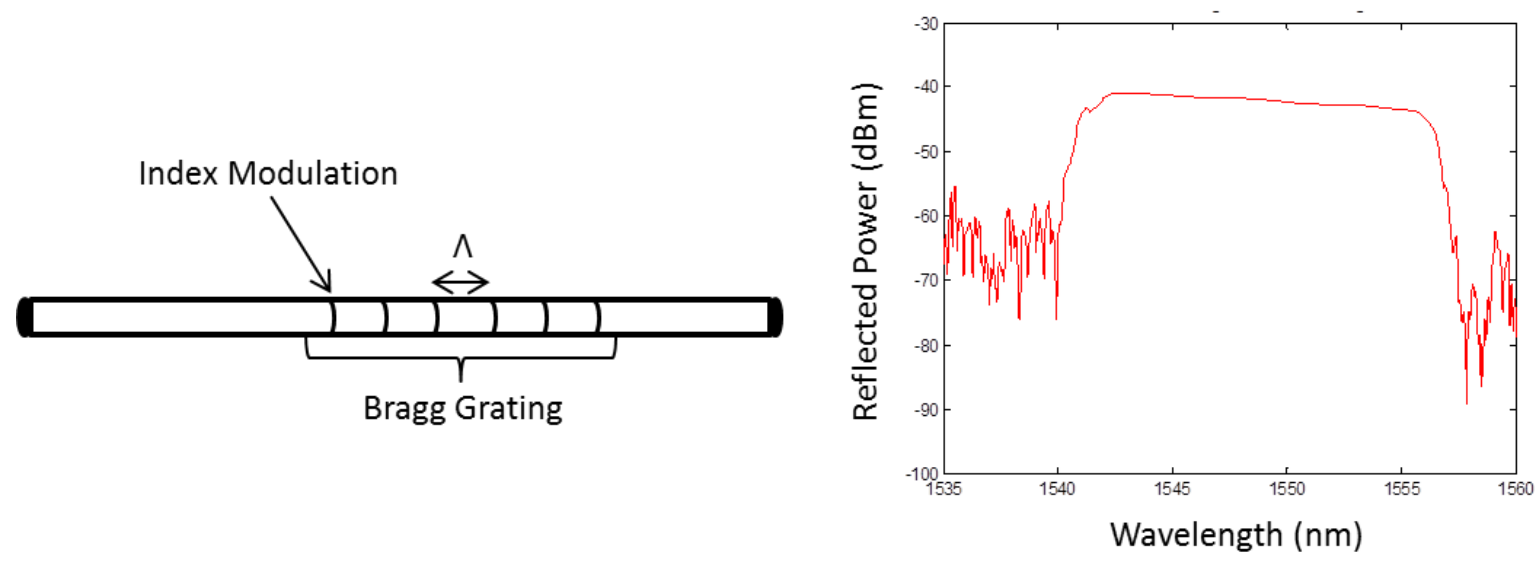

Figure 1. Overview of FBG Sensing and the effect on the transmitted spectrum

An inscribed FBG is a periodic modulation of the refractive index of the fibre core. As Figure 1 shows, this modulation produces a stop band in the transmission spectrum around a central wavelength, known as the Bragg wavelength. FBG sensing is typically done in a reflection configuration, where the grating produces a pass band around the Bragg wavelength, whose shape and power depend upon the characteristics of the grating inscription parameters. 


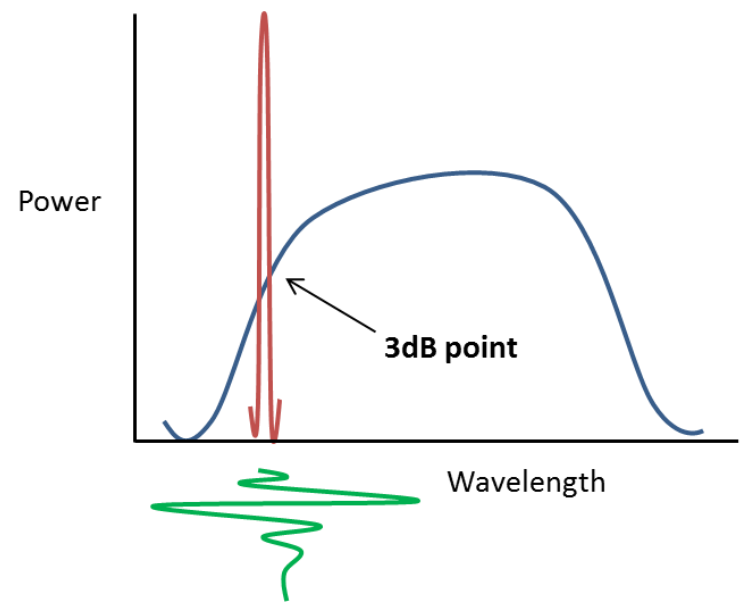

Figure 2. Sketch of a reflected spectrum profile of an FBG (blue) that is then used with a tuneable laser (red) and an incident ultrasonic wave (green) that induces a shift in the FBG profile

For ultrasonic measurements, first we analyse the profile of the grating in question, then tune a laser to the $3 \mathrm{~dB}$ point of the grating profile, stabilise the laser wavelength to compensate for low frequency drift observed at the detector and finally generate an incident ultrasonic wave. This causes a wavelength shift in the grating profile, producing an amplitude variation that can be observed and measured. Figure 2 shows the grating profile (blue) that has been examined and the $3 \mathrm{~dB}$ point located. A laser is then tuned to this wavelength (red) and the incident ultrasonic wave (green) creates a wavelength shift that is observed as a change in output power. For the results presented in the next section, the grating profile and the tuned laser profile can be observed in figure 3.

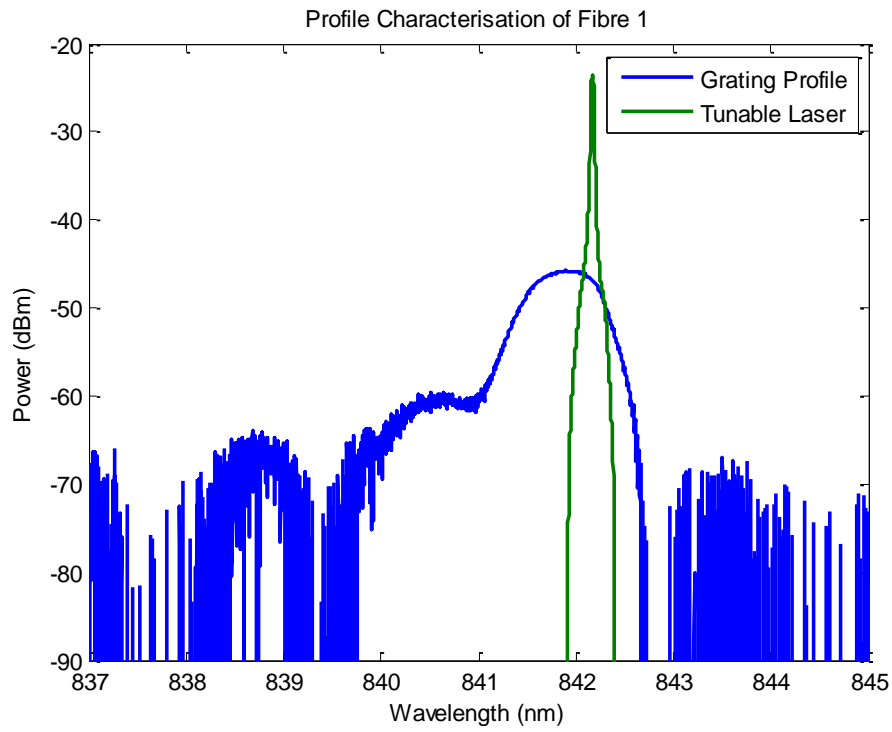

Figure 3. Depiction of characterised grating profile and laser tuned to the grating $3 \mathrm{~dB}$ point 
This analysis is undertaken using the setup shown in figure 4. Once the laser is tuned and then stabilised using piezo electrics, piezo electric transducers are positioned and acoustically coupled, using water as the acoustic medium.

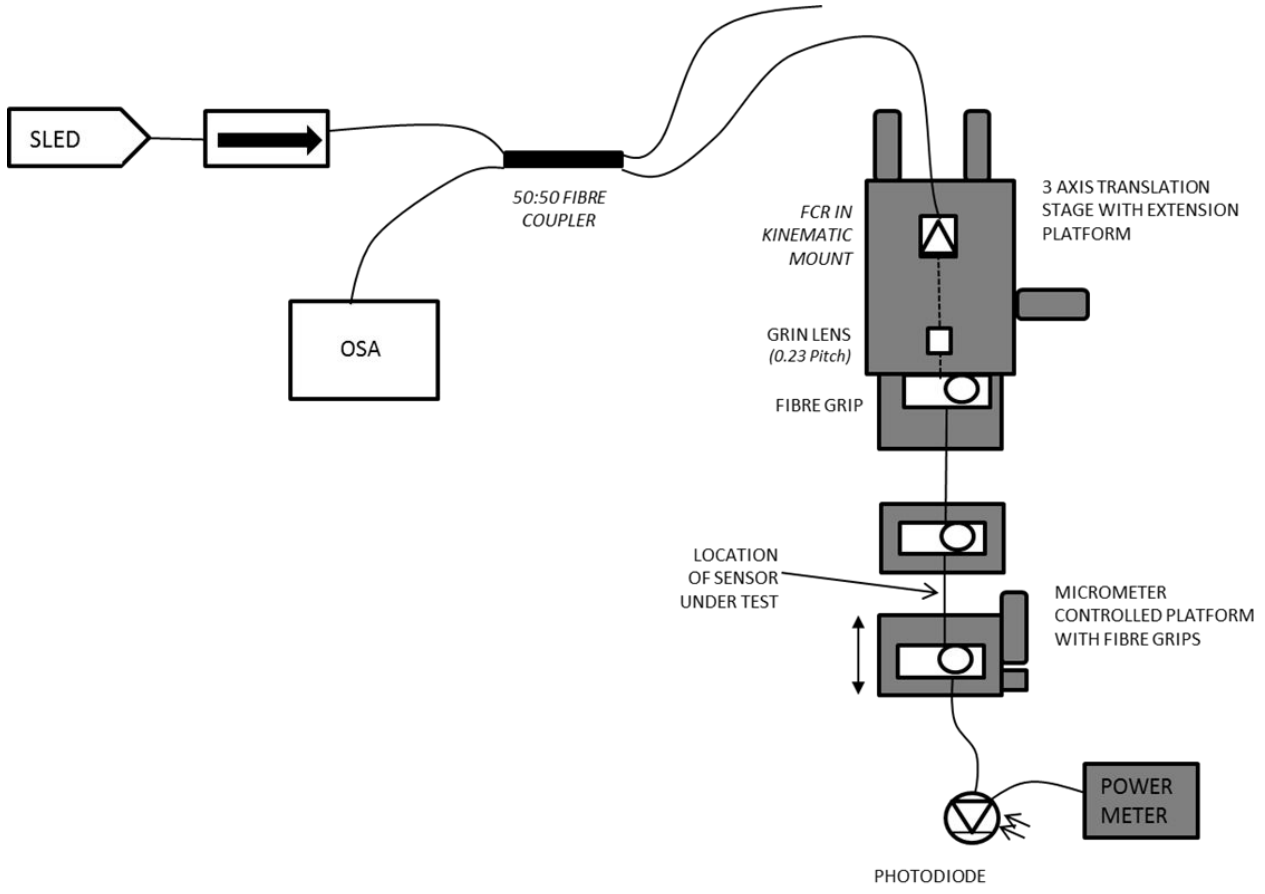

Figure 4. Schematic of the structure profile characterization setup

The profile characterisation setup is used as a base for the ultrasonic measurements to maintain coupling and to apply strain to the fibre if required. For ultrasonic measurements, the SLED and Isolator are replaced with an external cavity tuneable laser (Sacher, Lion TEC-500). 1MHz and 10MHz ultrasonic transducers (Panametrics V303 and V327) were excited using a square pulse generator (Panametrics 5077PR) and positioned in two discrete ways, as shown in figure 5.

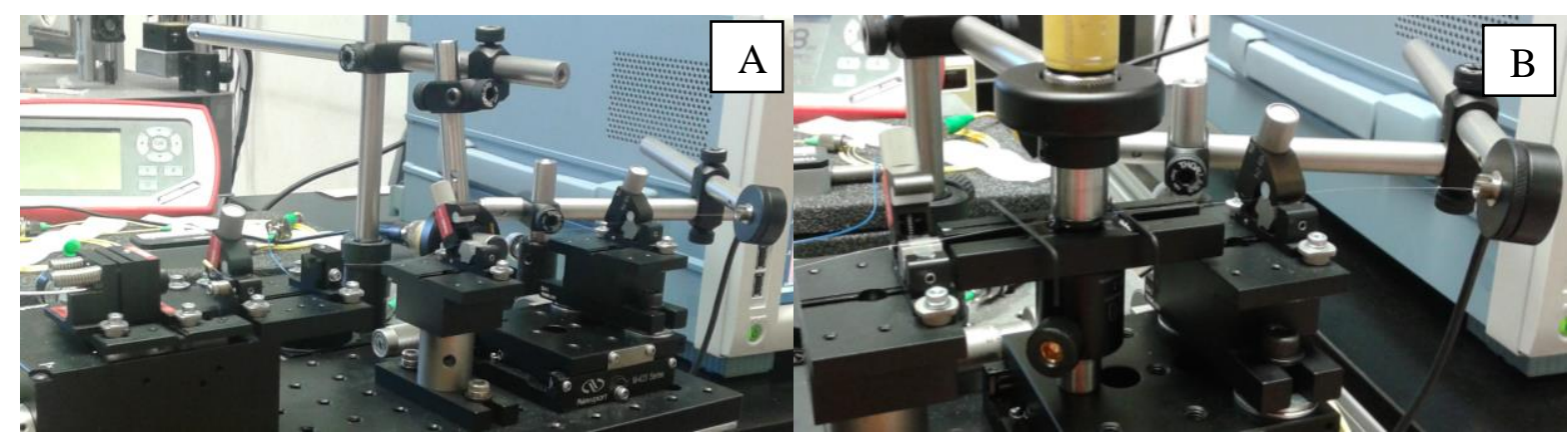

Figure 5. Ultrasonic detection configurations 1 and 2 as added to the profile characterisation setup in figure 4. A) Transducer underslung and pointing upwards towards fibre B) Transducer pointing vertically downwards, with fibre raised from supporting metal platform by allen keys.

Figure 5A shows the first configuration, where the transducer is positioned just below the fibre with water placed on the surface of the transducer for acoustic coupling. The fibre is secured in free space at the point of the grips. Figure 5B shows the second setup, with the fibre steadied with a metal platform and raised using allen keys. The transducer is positioned vertically over the fibre with water placed on the support for acoustic coupling. The second setup limits the movement of the fibre but provides the possibility of reflections from the support. 


\section{DISCUSSION OF RESULTS}

We have detected ultrasonic waves incident from $1 \mathrm{MHz}$ and $10 \mathrm{MHz}$ transducers using a PMMA mPOF Bragg grating with a central Bragg wavelength of $841 \mathrm{~nm}$ and a diameter of $125 \mu \mathrm{m}$. Figure 6 shows the results obtained.
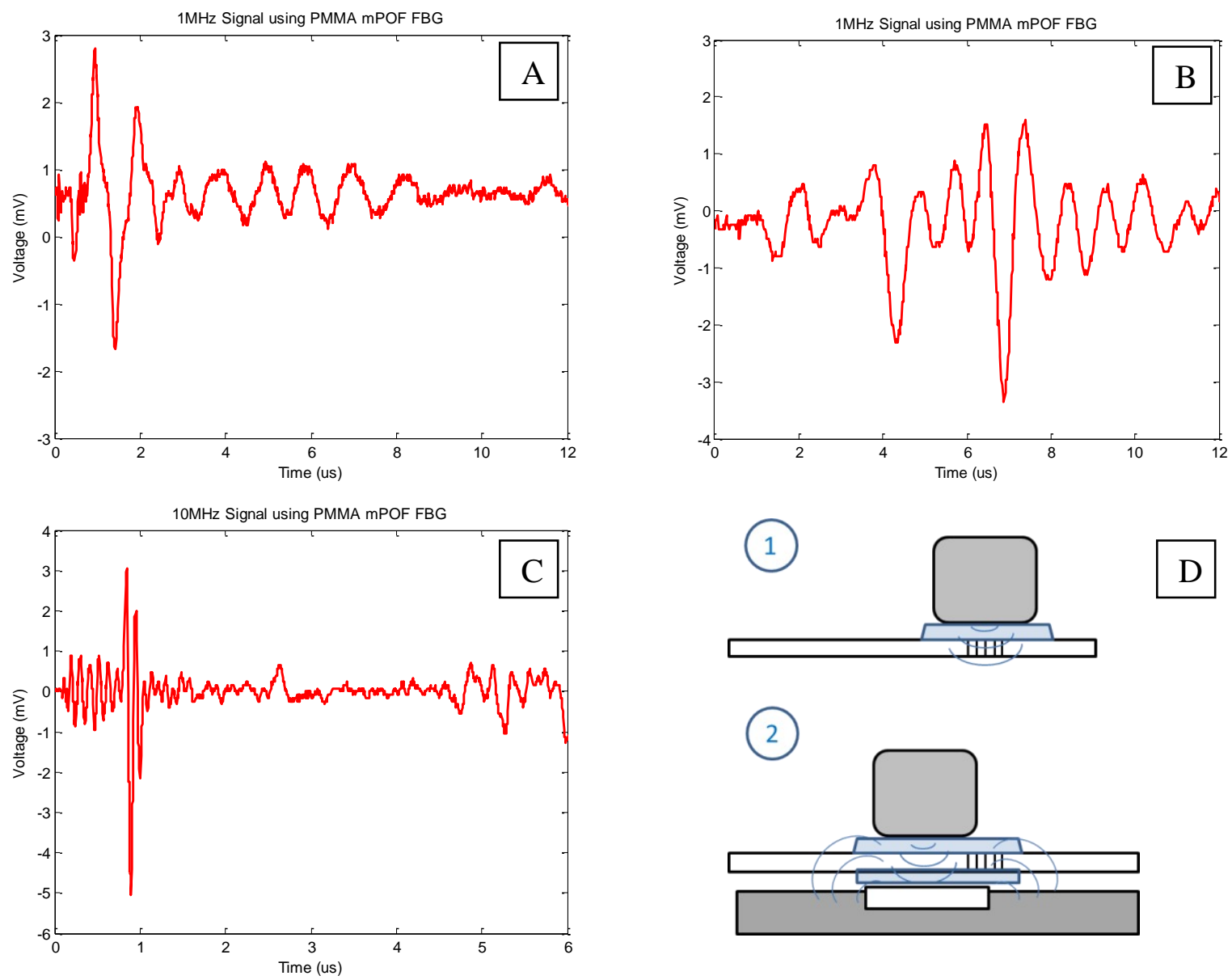

Figure 6. Ultrasonic detection using a PMMA mPOF FBG at $1 \mathrm{MHz}$ at $10 \mathrm{MHz}$. A) Signal received using ultrasonic setup 1 with a $1 \mathrm{MHz}$ transducer B) Signal received using ultrasonic setup 2 with a $1 \mathrm{MHz}$ transducer C) Signal received using ultrasonic setup 2 with a $10 \mathrm{MHz}$ transducer D) Diagram representation of setups 1 and 2 showing the transducer position, acoustic medium, fibre with FBG and support with screw hole

From the static appearance of figure 6A, the ultrasonic response could be perceived as positive. However, this signal varies over time in an interferometric manner with the expected performance of a Bragg grating being a response without a time variance. In order to clarify the issue, a change in setup was made from the configuration in figure 5A to figure 5B. This change introduced a metal support, raised the fibre and involved sweeping the transducer position to determine the effect of transducer position relative to the grating.

Figure 6B provides the answers by comparison with $6 \mathrm{~A}$. We can observe a number of effects, the first being a stable peak of low voltage which from the time delay can be said to be the direct detection of ultrasound from the grating, where the grating is not well aligned with the transducer. The second peak is of an interferometric nature and is comparably lower in amplitude and has an increased time delay. The interferometric signal is generated from a combination of the Bragg grating and one of the facets of the fibre. By manipulating the fibre after the Bragg grating and 
observing no effect, we concluded that the interferometric signal is generated between the input facet of the fibre and the Bragg grating itself. From the difference in amplitude of the interferometric signal, we can infer that there are a number of effects superimposed in figure 6A that are separated out in 6B. Two of these components are the detected ultrasonic response from the grating and the interferometric response between facet and grating.

We can observe two other effects of note in figure 6B. There is an overlap between the reverb of the outgoing pulse and the beginning of the following pulse, causing an increased amplitude visible after $8 \mu$ s in figure 6B. Comparison of all the figures in figure 6 show that at $10 \mathrm{MHz}$ the reverb is much smaller compared to the $1 \mathrm{MHz}$ result. Considering the inherent sensitivity of POF and considering the platform used as a support in setup 2 we have a number of detected ultrasonic waves with different time delays as can be observed in figure $6 \mathrm{~B}$ at $5 \mu$ s onwards. For instance, waves may reflect off the support and be detected at the grating. Given the depth of a screw hole in the support, we can identify that the signal received at $7 \mu \mathrm{s}$ is from this origin. Other displacements are the ultrasonic waves effectively coupling into the fibre and travelling to the grating.

There are several solutions to improving the quality of the detector along with several obstacles to be overcome. Solutions include precise positioning of the transducer to be over the grating, the removal of a support or the use of a uniform support to mitigate time-delayed reflections and acoustic shielding of the non FBG parts of the fibre. In order to remove or reduce the interferometric effects an angled fibre facet would be best, however difficulties providing consistent cleaving of POF will slow progress in this area. The best solution is to ensure that the surfaces are as perpendicular and uniform as possible given current cleaving methods and success rates, however further work may determine other methods. Connectorisation of the fibre may be an option as well, despite the high losses currently experienced.

\section{CONCLUSIONS AND FUTURE WORK}

We have presented a $125 \mu \mathrm{m}$ diameter $\mathrm{mPOF}$ Bragg grating ultrasound detector and observed the resultant signals using ultrasonic transducers at $1 \mathrm{MHz}$ and $10 \mathrm{MHz}$. We have analysed and discussed the different signals and effects, indicating potential methods to overcome the undesired responses. We have proved that POF Bragg gratings are capable of being used as ultrasound detectors with fibres that can fit inside a theoretical endoscope with a diameter of less than $1 \mathrm{~mm}$.

We conclude that this technology has implementation potential and that the issues discussed in the previous section need a solution that is viable. Given the fundamental acoustic sensitivity of PMMA mPOF and the suitable core diameter, the technology is favourable to provide a successful implementation when the different effects can be shielded out.

To further the development of this technology, elimination of unwanted signals needs to be achieved. The amplitude at the output needs to be improved, elimination of the interferometric component through modification of the facet, shielding of the fibre area to prevent acoustic coupling to the fibre and a different configuration to remove reflection from supports. From this point, good calibration can be achieved and then sensor positioning and topologies require study to determine a good detector setup. With these improvements, the final step is reducing the size of the detector to its minimum size.

\section{ACKNOWLEDGEMENTS}

The research leading to these results has received funding from the People Programme (Marie Curie Actions) of the European Union's Seventh Framework Programme FP7/2007-2013/ under REA grant agreement nº 608382.

We acknowledge and thank David Saez Rodriguez, who fabricated the PMMA fibres that were inscribed and characterised in this paper. 


\section{REFERENCES}

[1] L.H.V. Wang, Photoacoustic Imaging and Spectroscopy, $1^{\text {st }}$ ed., Optical Science and Engineering Series (CRC Press, 2009)

[2] K. Jansen, A. F. W. van der Steen, H. M. M. van Beusekom et al., "Intravascular photoacoustic imaging of human coronary atherosclerosis," Optics Letters, 36(5), 597-599 (2011).

[3] J.-M. Yang, C. Favazza, R. Chen et al., "Simultaneous functional photoacoustic and ultrasonic endoscopy of internal organs in vivo," Nature Medicine, 18(8), 1297 (2012).

[4] X. Bai, X. Gong, W. Hau et al, "Intravascular Optical-Resolution Photoacoustic

Tomography with a $1.1 \mathrm{~mm}$ Diameter Catheter" 20 March 2014 ( $8^{\text {th }}$ June 2015). http://www.ncbi.nlm.nih.gov/pmc/articles/PMC3961364/pdf/pone.0092463.pdf

[5] H. Lamela, D. Gallego and A. Oraevsky, "Optoacoustic Imaging using fiber-optic interferometric sensors", Optics Letters 34(23), 3695-3697 (2009).

[6] L. Bilro, N. Alberto, J. L. Pinto and R. Nogueira, "Optical Sensors Based on Plastic Fibers", Sensors 2012 ISSN 1424-8220 www.mdpi.com/journal/sensors

[7] K. Peters, "Polymer Optical Fibre Sensors - A Review", Smart Materials and Structures 2010

[8] D. Gallego, and H. Lamela, "High-sensitivity ultrasound interferometric single-mode polymer optical fiber sensors for biomedical applications," Optics Letters, 34(12), 1807-1809 (2009).

[9] D. Gallego, D. Saez Rodriguez, D. Webb et al, "Interferometric microstructured polymer optical fiber ultrasound sensor for optoacoustic endoscopic imaging in biomedical applications", Proc. of SPIE Vol. 9157 (2014) 